\title{
Plasmonic bacteria on a nanoporous mirror via hydrodynamic trapping for rapid identification of waterborne pathogens
}

Keumrai Whang ${ }^{1}$, Jong-Hwan Lee², Yonghee Shin' ${ }^{1}$ Wooju Lee ${ }^{3}$, Young Wan Kim³ ${ }^{3}$ Dongchoul Kim³ ${ }^{3}$ Luke P. Lee ${ }^{2}$ and Taewook Kang ${ }^{1}$

\begin{abstract}
A rapid, precise method for identifying waterborne pathogens is critically needed for effective disinfection and better treatment. However, conventional methods, such as culture-based counting, generally suffer from slow detection times and low sensitivities. Here, we developed a rapid detection method for tracing waterborne pathogens by an innovative optofluidic platform, a plasmonic bacteria on a nanoporous mirror, that allows effective hydrodynamic cell trapping, enrichment of pathogens, and optical signal amplifications. We designed and simulated the integrated optofluidic platform to maximize the enrichment of the bacteria and to align bacteria on the nanopores and plasmonic mirror via hydrodynamic cell trapping. Gold nanoparticles are self-assembled to form antenna arrays on the surface of bacteria, such as Escherichia coli and Pseudomonas aeruginosa, by replacing citrate with hydroxylamine hydrochloride in order to amplify the signal of the plasmonic optical array. Owing to the synergistic contributions of focused light via the nanopore geometry, self-assembled nanoplasmonic optical antennas on the surface of bacteria, and plasmonic mirror, we obtain a sensitivity of detecting $E$. coli as low as $10^{2}$ cells $/ \mathrm{ml}$ via surface-enhanced Raman spectroscopy. We believe that our label-free strategy via an integrated optofluidic platform will pave the way for the rapid, precise identification of various pathogens.
\end{abstract}

\section{Introduction}

Waterborne pathogen-related diseases are global health issues, leading to $>2.2$ million deaths per year (with 1.5 million of these deaths being reported for children) $)^{1,2}$. Conventional diagnostic techniques for waterborne pathogens, especially bacteria, are largely based on either selective culturing or molecular diagnosis, including immunoassays and the polymerase chain reaction. Although selective culturing has been considered the gold

Correspondence: Dongchoul Kim (dckim@sogang.ac.kr) or Luke P. Lee (Iplee@berkeley.edu) or Taewook Kang (twkang@sogang.ac.kr)

'Department of Chemical and Biomolecular Engineering, Sogang University, Seoul 04107, Korea

${ }^{2}$ Berkeley Sensor and Actuator Center, Departments of Bioengineering, Electrical Engineering and Computer Science, Biophysics Graduate Program, University of California, Berkeley, Berkeley, CA 94720, USA

Full list of author information is available at the end of the article.

These authors contributed equally: Keumrai Whang, Jong-Hwan Lee, Yonghee Shin standard for the identification of waterborne bacteria, this technique is limited by its time-consuming processes (typically taking 1-2 days for routine identification), lack of sensitivity and specificity, and difficulties related to cultures (sometimes nonculturable) ${ }^{3-6}$. Compared with selective culturing, molecular diagnosis exhibits better sensitivity and a faster detection time. However, this technique requires an additional sample preparation step to enrich the bacteria, which are dispersed in a large volume of water ${ }^{7}$. Moreover, this process still takes several hours and needs expensive reagents and equipment ${ }^{8,9}$.

On the other hand, nanostructure-based optical methods have gained increasing attention because of their high sensitivity and rapid detection time ${ }^{10-22}$. Among them, surface-enhanced Raman spectroscopy (SERS) is particularly attractive for the ultrasensitive detection of bacteria $^{17-22}$. Metallic nanostructures, including colloidal

\section{(c) The Author(s) 2018}

(c) (i) Open Access This article is licensed under a Creative Commons Attribution 4.0 International License, which permits use, sharing, adaptation, distribution and reproduction in any medium or format, as long as you give appropriate credit to the original author(s) and the source, provide a link to the Creative Commons license, and indicate if changes were made. The images or other third party material in this article are included in the article's Creative Commons license, unless indicated otherwise in a credit line to the material. If material is not included in the article's Creative Commons license and your intended use is not permitted by statutory regulation or exceeds the permitted use, you will need to obtain permission directly from the copyright holder. To view a copy of this license, visit http://creativecommons.org/licenses/by/4.0/. 
nanoparticles modified with specific antibodies or Raman active dyes or magnetic nanoparticles for the enrichment of bacteria, have also been demonstrated. However, the previously proposed SERS-based detection methods are generally limited to small sample volumes of a few microliters. Considering that waterborne bacteria are dispersed at very low concentrations, processing small sample volume undermines the reliability of detection. Therefore, besides high sensitivity and rapid detection time, an ideal SERS-based method for detecting waterborne bacteria should be capable of treating large sample volumes.

Here, we report plasmonic bacteria on a nanoporous mirror via hydrodynamic trapping, which allows enrichment of bacteria from large sample volumes and strong signal amplifications for the rapid identification of bacteria. Our design is schematically illustrated in Fig. 1. First, gold nanoparticles (GNPs) are spontaneously selfassembled to form antenna arrays on the surface of bacteria (plasmonic bacteria). Then, plasmonic bacteria are forced to be located on the nanopore of the membrane as a result of hydrodynamic trapping (Fig. 1a). Hydrodynamic trapping enables the enrichment of bacteria on the nanopore from large sample volumes. Once bacteria are trapped on the pore, owing to the synergistic contributions of (1) focused light by constructive interference between incident light and its diffraction via the nanopore, (2) self-assembled nanoplasmonic antennas on the surface of bacteria, and (3) plasmonic mirrors, a strong near-field enhancement between GNPs on plasmonic bacteria, as well as between GNPs on plasmonic bacteria with a gold thin mirror around the nanopore, (Fig. 1b) is expected. Sensitive label-free optical detection of bacteria from large sample volumes would therefore be possible with this design.

\section{Results}

For the self-assembly of GNPs on the bacterial surface, hydroxylamine hydrochloride (HAHC) is used to reduce the strongly negative surface charge of citrate-capped GNPs by replacing citrate ions with HAHC. Then, HAHC-modified GNPs with a diameter of $20 \mathrm{~nm}$ are mixed with Escherichia coli (E. coli). The representative scanning electron microscope (SEM) image in Fig. 2a, taken after mixing, shows that $E$. coli cells are densely covered by GNPs. To further verify the self-assembly of GNPs on the surface of $E$. coli, UV-vis spectra were measured before and after the mixing. Figure $2 b$ shows the absorbance spectra of an E. coli solution, HAHCmodified GNP solution, and mixed solution of E. coli and HAHC-modified GNPs. Before mixing GNPs with E. coli, the absorbance spectrum of the HAHC-modified GNP solution exhibits a single surface plasmon resonance (SPR) band at $522 \mathrm{~nm}$. After mixing, this SPR band is slightly redshifted $(\Delta \lambda=4 \mathrm{~nm})$, and another broad SPR band is observed in the near-infrared (NIR) region,

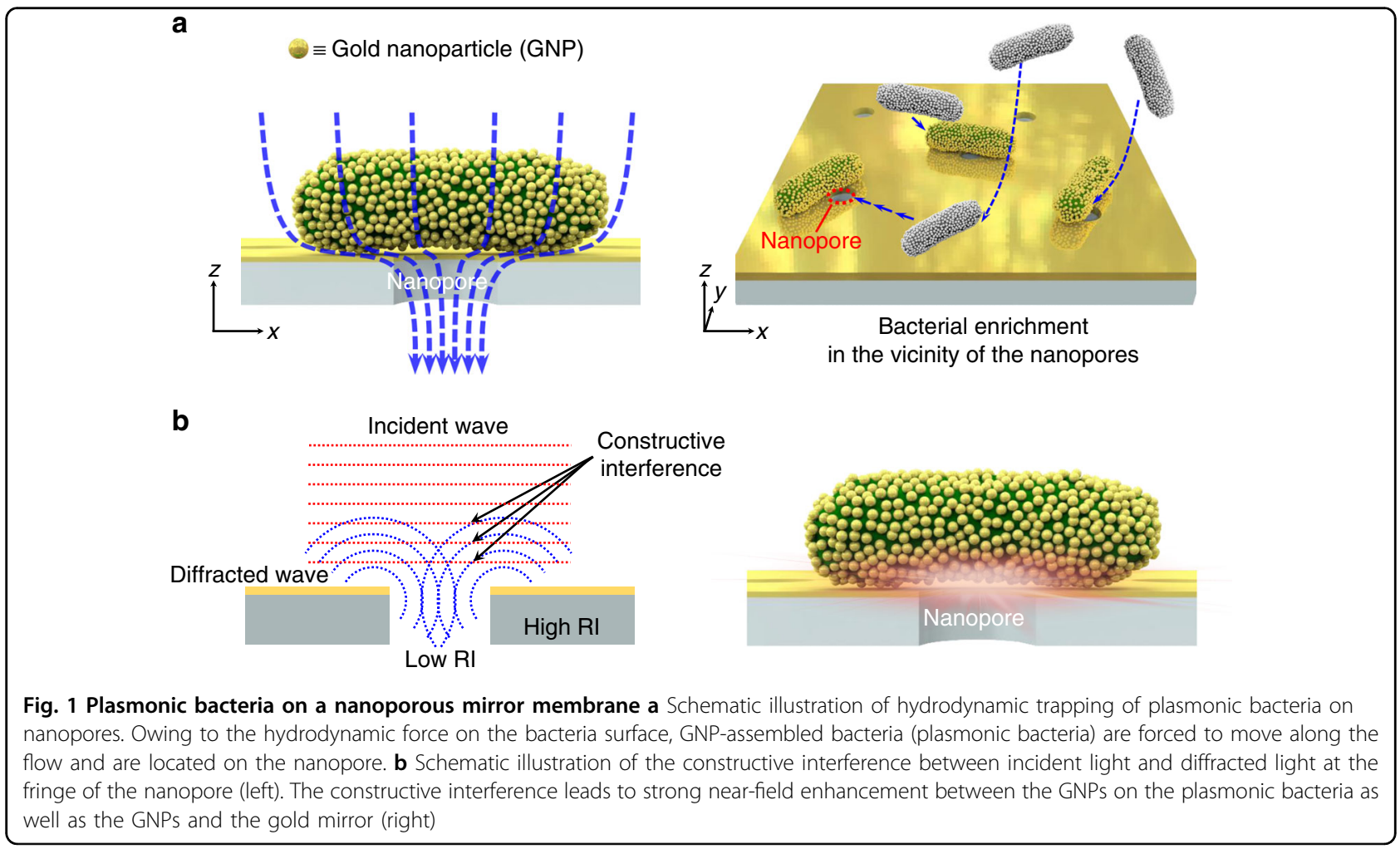




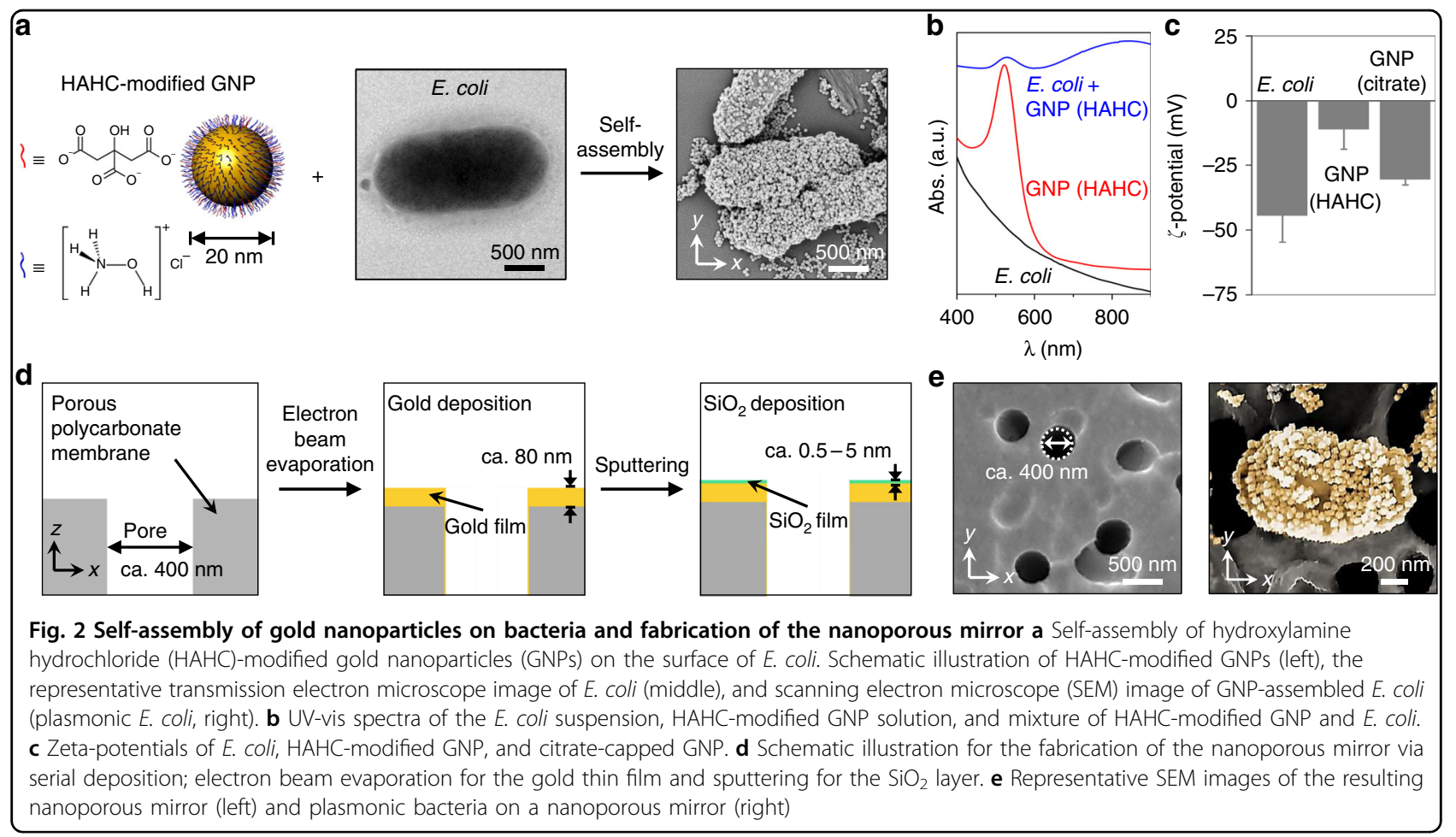

indicating that the surface plasmons of GNPs couple with each other. The decrease in the interparticle distance between GNPs when GNPs are self-assembled on the bacteria surface after mixing is mainly responsible for this observation. To examine the driving force for the selfassembly of GNPs, zeta-potentials and transmission electron microscope (TEM) measurements were carried out (Fig. 2c and Figure S1). The zeta-potentials of E. coli, HAHC-modified GNPs, and citrate-capped GNPs were found to be $-44.2 \pm 10.3,-10.9 \pm 7.8$, and $-30.3 \pm 2.2$ $\mathrm{mV}$, respectively. TEM images show that, unlike HAHCmodified GNPs, citrate-capped GNPs are not selfassembled on the surface of the bacteria. UV-vis spectra were also recorded before and after mixing citrate-capped GNPs with E. coli (Figure S2). The SPR band of citratecapped GNPs did not shift, and no additional SPR band was observed in the NIR region. From these results, it can be concluded that citrate-capped GNPs did not assemble on the surface of $E$. coli due to strong electrostatic repulsion. It is generally considered that nanoparticles are approximately neutral when their zeta-potentials are less than $\pm 10 \mathrm{mV}^{23}$. Therefore, the electrostatic repulsion between HAHC-modified GNPs and bacteria would be negligible. Therefore, hydrogen bonding between the hydroxyl group of hydroxylamine and the amine group of surface proteins or lipopolysaccharide ${ }^{24,25}$ would be responsible for the self-assembly of HAHC-modified GNPs on the E. coli surface. Note that HAHC-modified
GNPs also self-assemble to form dense arrays on the surface of Pseudomonas aeruginosa ( $P$. aeruginosa).

The nanoporous mirror for hydrodynamic cell trapping and signal amplifications was fabricated through successive depositions of metal and dielectric layers (Fig. 2d). First, an 80-nm-thick gold thin film was deposited on a porous polycarbonate $(\mathrm{PC})$ membrane with a pore diameter of $400 \mathrm{~nm}$ by electron beam evaporation. Subsequently, different thicknesses $(0.5,1,2$, and $5 \mathrm{~nm})$ of silicon dioxide $\left(\mathrm{SiO}_{2}\right)$ layers, which act as spacers between the gold thin film and plasmonic bacteria, were deposited over the gold thin film by radio frequency (RF) sputtering. The deposition of the gold thin film and $\mathrm{SiO}_{2}$ layers was confirmed by reflectance (Figure S3a). The gold thin film exhibited high reflectance of infrared or near-infrared wavelengths. Moreover, evaluating the reflectance spectra with respect to $\mathrm{SiO}_{2}$ thickness shows that the reflectance intensity for the gold film is inversely proportional to the $\mathrm{SiO}_{2}$ thickness. This proportionality is due to the $\mathrm{SiO}_{2}$ layer acting as an antireflective layer and decreasing the reflectivity with increasing thickness of the $\mathrm{SiO}_{2}$ layer $^{26}$. In addition, energy-dispersive X-ray spectroscopy (EDS) was carried out (Figure S3b). EDS elemental maps indicate that with the increase in $\mathrm{SiO}_{2}$ thickness, the $\mathrm{Si}$ atoms become more abundant on the surface of the membrane. The left image of Fig. 2e shows a representative SEM image of the resulting nanoporous mirror. Comparison with the SEM image of the bare porous PC membrane 


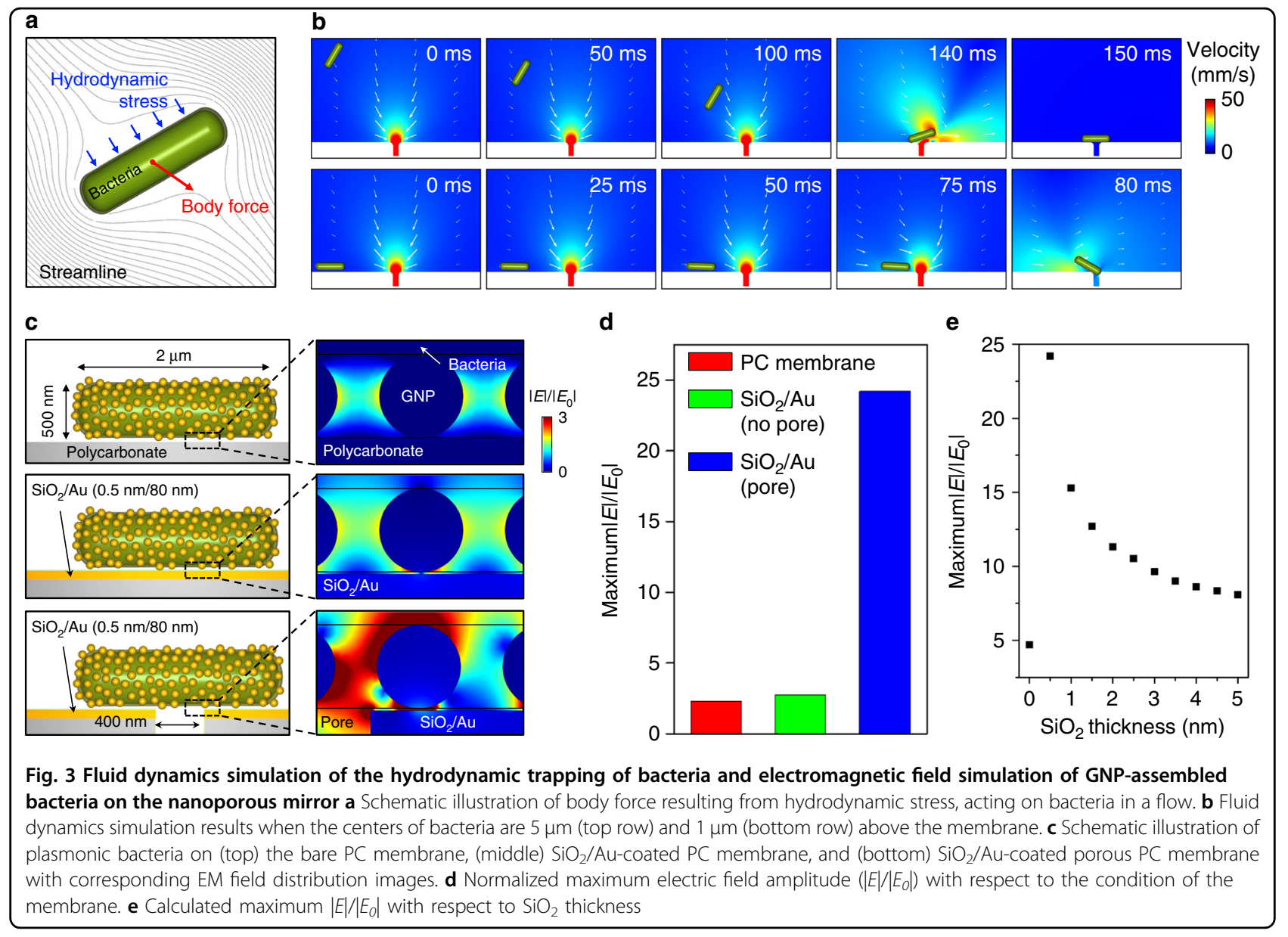

(before deposition) indicates that the pore size is slightly decreased by $3.7 \mathrm{~nm}$ (from $405.5 \pm 22.9 \mathrm{~nm}$ to $401.8 \pm$ $22.9 \mathrm{~nm}$, Figure S4). This result confirms that a structural change such as pore blocking does not occur during the deposition. A representative SEM image of the plasmonic bacteria on a nanoporous mirror after filtering a solution of plasmonic bacteria through the nanoporous mirror is shown in the right image of Fig. 2e (a low-magnification image is also shown in Figure S5).

A two-dimensional fluid dynamics simulation was carried out for the hydrodynamic trapping of bacteria on the nanopores (Fig. 3a). Bacteria are assumed to be a rod with a diameter of $500 \mathrm{~nm}$ and a length of $2 \mu \mathrm{m}$. To observe the effect of the pore on the hydrodynamic trapping, the effect of fluid flow resulting from numerous pores and the wall of the fluidic channel is assumed to be negligible, and a single pore with a diameter of $400 \mathrm{~nm}$ in a large fluidic channel (diameter of $100 \mu \mathrm{m}$ ) is considered. The fluid flow and the concomitant movement of bacteria are calculated simultaneously by using a fluid structure interaction (FSI) method ${ }^{27}$. In this method, the incompressible Navier-Stokes equation is solved to calculate the fluid flow. At the same time, hydrodynamic stress on bacteria
$(\Gamma)$ is calculated to predict the movement of bacteria in a flow using the following equation,

$$
\Gamma=\left[-p I+\mu\left(\nabla u_{\text {fluid }}+\left(u_{\text {fluid }}\right)^{T}\right)\right]
$$

where $u_{\text {fluid }}, p$, and $\mu$ are the fluid velocity, pressure, and kinematic viscosity, respectively. Finally, the body force $(F)$ resulting in the movement of bacteria is calculated by the following equation,

$$
F=\int_{V} \nabla \cdot \sigma d V
$$

where $d V$ is an infinitesimal volume element and $\sigma$ is a stress field. The stress at the surface of bacteria satisfies the boundary condition $\sigma \cdot n=\Gamma \cdot n$, where $n$ is a normal vector to the surface of bacteria.

Time sequence images of bacteria when the centers of bacteria are initially $5 \mu \mathrm{m}$ (top row) and $1 \mu \mathrm{m}$ (bottom row) above the membrane surface are shown in Fig. 3b. In both cases, owing to the hydrodynamic pressure acting on the bacteria surface, bacteria are forced to move along the flow and finally trapped on the nanopore. Irrespective of 
the initial location of bacteria relative to the nanopore (i.e., different orientation and distance), bacteria are found to be trapped on the pore in the end (Figure S6, Supplementary Movie 1, and Supplementary Movie 2).

To estimate the optical amplifications of the plasmonic bacteria on a nanoporous mirror, an electromagnetic (EM) simulation was conducted. Figure 3c shows the simulation schemes for plasmonic bacteria on different membranes ( $\mathrm{PC}$ membrane without nanopores, $\mathrm{SiO}_{2}$ and gold thin films $\left(\mathrm{SiO}_{2} / \mathrm{Au}\right)$ on the $\mathrm{PC}$ membrane without nanopores, and the nanoporous mirror) and their EM field distributions (the dotted square box in each scheme). The dimensions of bacteria are identical to those used for the fluid dynamics simulation. The diameter of the GNP on plasmonic bacteria is fixed at $20 \mathrm{~nm}$. The thicknesses of $\mathrm{SiO}_{2}$ and gold films are 0.5 and $80 \mathrm{~nm}$, respectively. The circular pore diameter is fixed at $400 \mathrm{~nm}$ in accordance with our experimental conditions. In the EM simulation, the local electric field enhancement $\left(|E| /\left|E_{0}\right|\right)$ from the ratio of the near field $(|E|)$ and the incident field $\left(\left|E_{0}\right|\right)$ is calculated. The EM field distribution images indicate that when $\mathrm{SiO}_{2}$ and $\mathrm{Au}$ are deposited on the membrane, the local electric field enhancement increases, particularly in the space between $\mathrm{SiO}_{2} / \mathrm{Au}$ and the GNPs because of the strong coupling between the two surface plasmons (i.e., the GNPs and gold film). Interestingly, with the presence of the nanopores, the field enhancement significantly increases. As nanopores are known to act as a diffraction grating at the fringe of the pore ${ }^{28-30}$, constructive interference between incident light and its diffraction can induce field enhancement. This possibility is also supported by the field enhancement observed in the lowmagnification EM field distribution images in Figure S7.

The EM simulation results were quantitatively analyzed for the comparison of the maxima of the local electric field enhancement in the space between bacteria and the membrane for each case (Fig. 3d). The maximum local electric field enhancement values were found to be 2.33, 2.77 , and 24.20 , respectively. This result indicates that the presence of both the nanopore and $\mathrm{SiO}_{2} / \mathrm{Au}$ on the membrane increase the local field enhancement 10.4 times. On the other hand, owing to the contribution of the nanopore alone, the local field enhancement is estimated to be increased 8.7 times. These results suggest that in our design, the contribution of the constructive interference to the electric field amplification would be comparable to that of the plasmon coupling between the GNPs and the gold film. According to our fluid dynamics simulation, most bacteria are expected to be located on the nanopores during the filtration. Therefore, it is reasonable to assume that in our design, the optical signal of the plasmonic bacteria on a nanoporous mirror would originate mainly from the nanopore. An EM simulation of a similar design without GNP was also conducted (Figure S8). The effect of GNPs on the local electric field enhancement was maximized when the nanoporous mirror was used.

The near-field enhancement between the GNPs and the gold film is known to be affected by the $\mathrm{SiO}_{2}$ thickness ${ }^{31}$. To examine the effect of $\mathrm{SiO}_{2}$ thickness, an EM simulation was also carried out with different $\mathrm{SiO}_{2}$ film thicknesses (from 0 to $5.0 \mathrm{~nm}$ ). Figure 3e shows the maxima of $|E| /\left|E_{O}\right|$ with respect to the $\mathrm{SiO}_{2}$ thickness. Figure $\mathrm{S} 9$ also shows the EM field distribution images. When decreases in the thickness of $\mathrm{SiO}_{2}$ from $5 \mathrm{~nm}$ to $0.5 \mathrm{~nm}$, the maximum local electric field enhancement exponentially increases. This change is related to the distancedependent weakening of the plasmon coupling between the GNPs and gold film. Note that the dramatic decrease in the near-field enhancement in the absence of the $\mathrm{SiO}_{2}$ film is related to the formation of a contact where electric conduction occurs. The electric conduction significantly weakens the strength of the plasmon coupling ${ }^{32-34}$. In addition, we have investigated how the maximum of electric field enhancement varies with changes in the wavelength of the incident light by EM simulation. As shown in Figure S10, the maxima of the electric field enhancement increased as the thickness of $\mathrm{SiO}_{2}$ decreased from 5 to $0.5 \mathrm{~nm}$. No noticeable shift was observed, irrespective of the wavelength of the incident light.

To realize the benefits of plasmonic bacteria on a nanoporous mirror, this technique was applied to the detection of E. coli and P. aeruginosa via SERS. These bacteria were selected as they are involved in most waterborne diseases. First, plasmonic bacteria were quickly prepared and filtered through the nanoporous mirror. Then, Raman spectra were obtained from the membrane surface. Fig. 4a shows the representative Raman spectra of two bacteria on the surface of the membrane. Three Raman transitions for both bacteria are commonly observed at $717 \mathrm{~cm}^{-1}, 958 \mathrm{~cm}^{-1}$, and 1351 $\mathrm{cm}^{-1}$, which correspond to a glycosidic ring mode, $v(\mathrm{CN})$, and $v\left(\mathrm{COO}^{-}\right)$, respectively ${ }^{17-20}$. Note that the structure of the cell membrane that is responsible for the observed SERS signals is similar for both bacteria as they are gramnegative ${ }^{35}$. Distinctive Raman transitions of E. coli and $P$. aeruginosa are also found at $1312 \mathrm{~cm}^{-1}$ and $1155 \mathrm{~cm}^{-1}$, respectively. These Raman transitions can be assigned to $v$ $\left(\mathrm{NH}_{2}\right)$ of adenine ${ }^{20}$ for $E$. coli and $\omega\left(\mathrm{N}-\mathrm{CH}_{3}\right)$ of pyocya$\operatorname{nin}^{36}$ for $P$. aeruginosa.

To further elucidate the effects of the GNPs, mirror, and nanopores on the observed SERS enhancement, bare E. coli (E. coli without GNPs) and GNP-assembled E. coli (i.e., plasmonic $E$. coli) were filtered through the nanoporous mirror and porous PC membrane, respectively. Raman spectra were measured. As shown in Fig. 4b, Raman transitions that are apparent for the plasmonic $E$. coli on the nanoporous mirror are not observed in either case (i.e., bare $E$. coli on the nanoporous mirror and 


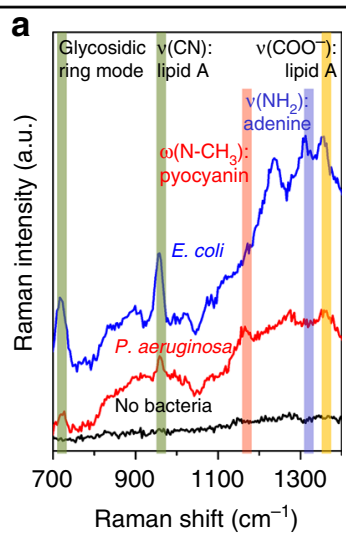

c

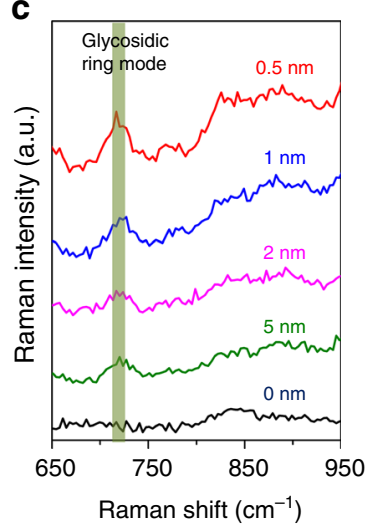

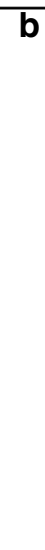

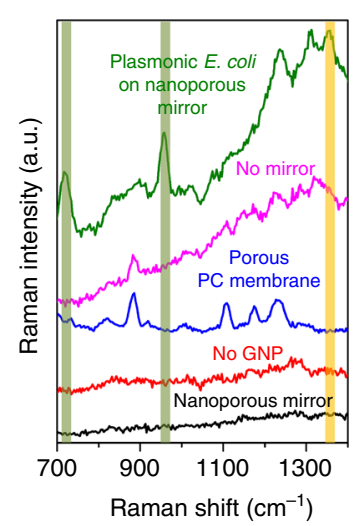

d

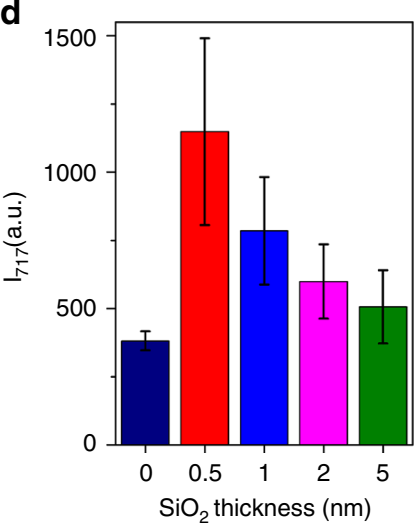

e
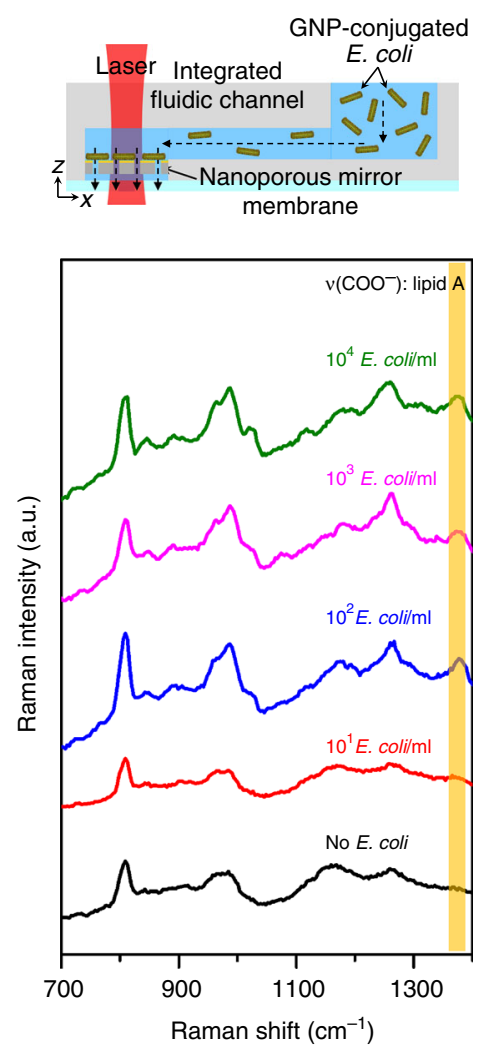

Fig. 4 Raman measurement of plasmonic bacteria on nanoporous mirror a Raman spectra of nanoporous mirror with no bacteria (black), plasmonic $P$. aeruginosa on the nanoporous mirror (red), and plasmonic E. coli on the nanoporous mirror (blue). Green and yellow boxes indicate the common Raman transitions of E. coli and P. aeruginosa. Red and blue boxes indicate the distinctive Raman transitions of $P$. aeruginosa and $E$. coli, respectively. $\mathbf{b}$ Raman spectra of the nanoporous mirror with no bacteria (black), bare E. coli on the nanoporous mirror (no GNPs, red), the porous PC membrane (blue), plasmonic E. coli on the porous PC membrane (magenta) and plasmonic E. coli on the nanoporous mirror (green). c Raman spectra of plasmonic $E$. coli on the membrane with different $\mathrm{SiO}_{2}$ thicknesses (from the top, $0.5,1,2,5$, and $0 \mathrm{~nm}$ ). $\mathbf{d}$ Raman intensity at $717 \mathrm{~cm}^{-1}$ with respect to $\mathrm{SiO}_{2}$ thickness. e Schematic illustration of the nanoporous mirror-integrated fluidic channel and Raman spectra measured after filtering $1 \mathrm{ml}$ of plasmonic E. coli solution through the channel

plasmonic E. coli on the porous PC membrane). These results strongly imply that the nanoporous mirror or GNPs do not induce strong SERS enhancement but, instead, the synergistic contributions of the plasmonic bacteria on a nanoporous mirror is mainly responsible for the observed SERS enhancement.

To compare the signal enhancement with respect to $\mathrm{SiO}_{2}$ thickness with the simulation result, a solution of GNP-assembled $E$. coli was filtered by the nanoporous mirrors with different $\mathrm{SiO}_{2}$ thicknesses, and Raman spectra were obtained (Fig. 4c). Similar to the EM simulation result, the intensity at $717 \mathrm{~cm}^{-1}$ increased as the $\mathrm{SiO}_{2}$ thickness decreased (Fig. 4d). No Raman transition peak was observed from the membrane without a $\mathrm{SiO}_{2}$ layer.

To investigate the sensitivity and detection time of our design, the plasmonic bacteria on a nanoporous mirror were further integrated into a simple fluidic channel. The fact that most bacteria are enriched on the nanoporous mirror was also confirmed by culturing the suspensions of bacteria before and after filtration (Figure S11). After the conjugation of GNPs onto E. coli, $1 \mathrm{ml}$ of plasmonic E. coli solution was passed via the integrated fluidic channel, and Raman signal was directly measured. Figure $4 \mathrm{e}$ shows the Raman spectra at different concentrations of E. coli. All Raman spectra were analyzed on the basis of the Raman transition of $v\left(\mathrm{COO}^{-}\right)$because the other Raman peaks show either weak intensity or strong interference with the Raman peaks of the fluidic channel materials (e.g., poly (methyl methacrylate), PMMA). From the Raman transition of $v\left(\mathrm{COO}^{-}\right)$, the limit of detection is found to be $10^{2}$ cells $/ \mathrm{ml}$. To examine the reproducibility of the SERS signal, SERS signals at four random spots were measured after filtration (spot size of laser: $0.2 \mathrm{~mm}$, Figure S12a). Then, the average and standard deviation of the normalized Raman intensity of PMMA $\left(987 \mathrm{~cm}^{-1}, \mathrm{C}-\mathrm{C}\right.$ stretching) were calculated (Figure S12b). The average and standard deviation calculated after filtering $10^{2} \sim 10^{4}$ 
E. coli $/ \mathrm{ml}$ are $0.85 \pm 0.02,0.81 \pm 0.08$, and $0.92 \pm 0.04$, respectively. The standard deviation values indicate that the SERS signals from the plasmonic bacteria on a nanoporous membrane are quite reproducible. Note that a PC membrane with a smaller size $(4 \mathrm{~mm}$ in diameter in our experiment) would slightly improve the reliability and reproducibility of the SERS signals. To evaluate the quantification of our method, the Raman transition of bacteria at $1351 \mathrm{~cm}^{-1}$, which can be assigned to $v\left(\mathrm{COO}^{-}\right)$ of lipid A, was normalized to 4 different Raman transitions of PMMA as internal standards $\left(600 \mathrm{~cm}^{-1}, 812\right.$ $\mathrm{cm}^{-1}, 964 \mathrm{~cm}^{-1}$, and $1448 \mathrm{~cm}^{-1}$, corresponding to $v$ $(\mathrm{O}-\mathrm{C}=\mathrm{O}), v(\mathrm{C}-\mathrm{C}-\mathrm{C}-\mathrm{C}), v(\mathrm{C}-\mathrm{C})$, and $v\left(\mathrm{CH}_{2}\right)$, respectively $)^{37}$. As shown in Figure S13, the normalized Raman intensities generally increase with increasing concentration of bacteria, regardless of which Raman peak of PMMA is used as an internal standard. Regarding the detection time of our integrated platform, each step, that is, the conjugation between the GNPs and bacteria (i.e., the formation of plasmonic bacteria), the enrichment, and the detection, takes $\sim 9 \mathrm{~min}, 30 \mathrm{~s}$, and $5 \mathrm{~s}$, respectively. The total detection time is shorter than $c a .10 \mathrm{~min}$. Note that this detection time could be shortened by improving the conjugation time.

\section{Discussion}

In conclusion, we have designed an integrated platform of a plasmonic bacteria on nanoporous mirror membrane via hydrodynamic trapping for the enrichment of bacteria and strong signal amplifications. HAHC-modified GNPs spontaneously self-assembled to form dense arrays on the surface of bacteria, such as $E$. coli and $P$. aeruginosa, in order to amplify the optical signal. Fluid dynamics simulations reveal that bacteria are trapped on the nanopore during filtration, resulting in the enrichment. EM simulations show that, owing to the nanopore geometry, the electric field is enhanced $>10$ times compared with that without the nanopores. The near-field amplifications of our design can be attributed to (1) the focused light by constructive interference between incident light and its diffracted one via the nanopore geometry, (2) selfassembled nanoplasmonic optical antennas on the surface of the bacteria, and (3) the plasmonic mirror. Our plasmonic bacteria on a nanoporous mirror platform was successfully applied to the detection of E. coli and $P$. aeruginosa. Through further integration into a fluidic channel, trace $E$. coli concentrations as low as $10^{2}$ cells $/ \mathrm{ml}$ are detectable via SERS. We believe that our integrated optofluidic platform paves the way for the rapid, precise identification of various pathogens and further understanding of microbial resistance for effective treatment as the platform can provide insights regarding microbial surface expression to correlate with the genome of emerging pathogens and the effects of the water surface and the environmental impacts of the transmission line.

\section{Materials and methods Synthesis of HAHC-modified GNPs}

GNPs modified with HAHC were synthesized based on the previously reported method ${ }^{38}$. In brief, for the synthesis of seed particles, $125 \mathrm{ml}$ of $254 \mu \mathrm{M}$ $\mathrm{HAuCl} \cdot 3 \mathrm{H}_{2} \mathrm{O}$ solution was prepared with deionized (DI) water and boiled in a $250 \mathrm{ml}$ round-bottom flask in an oil bath at reflux with vigorous stirring. In addition, $12.5 \mathrm{ml}$ of $40 \mathrm{mM}$ trisodium citrate dehydrate prepared with DI water was rapidly added to the flask with continuous vigorous stirring for $10 \mathrm{~min}$, and the solution was stirred for $15 \mathrm{~min}$ at room temperature (RT). For the growth and replacement of citrate with HAHC, $30 \mathrm{ml}$ of seed particle solution and $3 \mathrm{ml}$ of $0.2 \mathrm{M} \mathrm{HAHC}$ were subsequently added to $270 \mathrm{ml}$ of DI water with vigorous stirring at RT. Then, $2.5 \mathrm{ml}$ of $25.4 \mathrm{mM} \mathrm{HAuCl} 4 \cdot 3 \mathrm{H}_{2} \mathrm{O}$ solution was added dropwise to the mixture for $1 \mathrm{~min}$.

\section{Fabrication of the nanoporous mirror}

The nanoporous mirror was fabricated through two steps of serial deposition of a gold $(\mathrm{Au})$ thin film and a $\mathrm{SiO}_{2}$ layer. First, the PC porous membrane (Cyclopore 400-nm pore size, GE Healthcare Bio-Sciences, PA, USA) was loaded in an electron beam evaporation for the gold thin film deposition. A 5-nm-thick titanium layer, as an adhesion layer, and an 80-nm-thick $\mathrm{Au}$ film were sequentially deposited on the $\mathrm{PC}$ porous membrane under a base pressure of $2 \times 10^{-7}$ Torr. Afterwards, $\mathrm{SiO}_{2}$ layers with different thicknesses $(0.5,1,2$, and $5 \mathrm{~nm})$ were deposited over the thin Au film by RF sputtering. The reflectance spectra of the nanoporous mirrors were measured using a UV-VIS-NIR scanning spectrophotometer (UV-3101PC, Shimadzu, MD, USA), and the EDS was carried out for the elemental mapping of $\mathrm{Si}$ atoms using an EDS attached to the SEM/FIB (Quanta 3D FEG, OR, USA).

\section{Fluid dynamics simulation}

For the examination of the hydrodynamic trapping of bacteria on nanopore, the fluid flow and the movement of bacteria were calculated simultaneously by using a FSI method $^{27}$. Bacteria was assumed to have a rod shape with a diameter of $500 \mathrm{~nm}$ and a length of $2 \mu \mathrm{m}$. The membrane was assumed to have a single pore with a diameter of $400 \mathrm{~nm}$ in a chamber with a diameter of $100 \mu \mathrm{m}$.

\section{EM simulation}

To investigate the optical properties of GNP-assembled bacteria (plasmonic bacteria) on the nanoporous mirror, wave optics simulations were carried out using the 
commercial EM simulation package (COMSOL Multiphysics). The complex values of the wavelengthdependent refractive index of bulk gold were taken from the database reported by Johnson and Christy ${ }^{39}$. The refractive index values of $\mathrm{SiO}_{2}$ and $\mathrm{PC}$ were set to be 1.45 and 1.56, respectively. The $p$-polarized EM light was incident to the surface of the nanoporous membrane. The wavelength of incident light was set to be $785 \mathrm{~nm}$. In this simulation, we assumed that the GNPs attached on the surface of bacteria were uniformly distributed with an interparticle distance of $10 \mathrm{~nm}$.

\section{Bacterial growth}

E. coli and $P$. aeruginosa were grown in Lysogeny broth medium at $37^{\circ} \mathrm{C}$ with shaking $(250 \mathrm{ml}$ Erlenmeyer flasks, $150 \mathrm{rpm}$ ). When the culture turbidity (OD 600) reached 1.0, bacterial cells were harvested by centrifugation (4500 rpm, $10 \mathrm{~min}$ ), and E. coli and P. aeruginosa cell pellets were suspended in DI water, followed by three washes with DI water for evaluating the SERS application.

\section{Design and fabrication of integrated fluidic channel}

The integrated fluidic channel was designed as shown in Figure S14. The diameter and the height of the detection zone were $4 \mathrm{~mm}$ and $0.28 \mathrm{~mm}$, respectively. To integrate the channel into a disposable cartridge, PMMA sheets and PC sheets were used for the top and bottom layers, respectively. Top and bottom layers were cut with a VersaLASER VL-200 laser cutting system (Universal Laser System, Inc., Scottsdale, AZ, USA). All samples were cleaned with $70 \%$ ethanol twice for $10 \mathrm{~min}$, rinsed with DI water, and dried using $\mathrm{N}_{2}$. Then, the top and bottom layers were bonded together using $80 \mu \mathrm{m}$-thick doublesided tape (ARcare ${ }^{\circledast}$ 90445, Adhesives Research, Inc., Glen Rock, PA, USA). To load the sample using a syringe, a loading zone was made of polydimethylsiloxane (PDMS) and introduced at the top layer of the fluidic channel. Finally, the nanoporous mirror was bonded to the bottom region of the fluidic channel with the double-side tape.

\section{Raman measurement}

For Raman measurement, a commercial Raman spectrometer (QE65000 from Ocean Optics Inc.) and a $785 \mathrm{~nm}$ laser module (I0785MM0350MS from Innovative Photonic Solution Inc.) were used. A $785 \mathrm{~nm}$ laser operated at a power of $250 \mathrm{~mW}$ was used in the Raman measurements with $5 \mathrm{~s}$ of integration time.

\section{Acknowledgements}

This work was supported by the Air Force Office of Scientific Research Grants AFOSR FA2386-13-1-4120, by the International Research and Development Program of the National Research Foundation of Korea (NRF), by the Ministry of Science, ICT, and Future Planning (MSIP) (no. 2016K1A3A1A32913356), by the Mid-Career Researcher Support Program of NRF by the MSIP (no.
2016R1A2B3014157), by the Basic Science Research Program through the NRF funded by the Ministry of Education (no. 2016R1A6A1A03012845), by the C1 Gas Refinery Program of NRF by the MSIP (no. 2016M3D3A1 A01913546), by the Leading Foreign Research Institute Recruitment Program through NRF by the MSIP (no. 2013K1A4A3055268), and by the National Institutes of Health (NIH) (R01 Al117064-01).

\section{Author details}

'Department of Chemical and Biomolecular Engineering, Sogang University, Seoul 04107, Korea. ${ }^{2}$ Berkeley Sensor and Actuator Center, Departments of Bioengineering, Electrical Engineering and Computer Science, Biophysics Graduate Program, University of California, Berkeley, Berkeley, CA 94720, USA. ${ }^{3}$ Department of Mechanical Engineering, Sogang University, Seoul 04107, Korea

\section{Author contributions}

L.P.L. and T.K. conceived the concept. K.W., J.-H.L., Y.S., and T.K. designed and organized the experiments. K.W. and J.-H.L. performed the experiments. W.L., Y.W.K., and D.K. contributed to the simulations. K.W., J.-H.L., W.L., Y.W.K., L.P.L., and T.K. wrote the manuscript. All authors discussed the results and commented on the manuscript.

\section{Conflict of interest}

The authors declare that they have no conflict of interest

Supplementary information is available for this paper at https:/doi.org/ 10.1038/s41377-018-0071-4.

Received: 7 March 2018 Revised: 24 August 2018 Accepted: 9 September 2018 Accepted article preview online: 12 September 2018

Published online: 03 October 2018

\section{References}

1. World Health Organization (WHO). Water sanitation and health. http://www. who.int/water_sanitation_health/en/ (2015).

2. Ramirez-Castillo, F. Y. et al. Waterborne pathogens: detection methods and challenges. Pathogens 4, 307-334 (2015).

3. Zhao, X. H., Lin, C. W., Wang, J. \& Oh, D. H. Advances in rapid detection methods for foodborne pathogens. J. Microbiol. Biotechnol. 24, 297-312 (2014).

4. Law, J. W. F., Ab Mutalib, N. S., Chan, G. K. \& Lee, L. H. Rapid methods for the detection of foodborne bacterial pathogens: principles, applications, advantages and limitations. Front. Microbiol. 5, 770 (2015).

5. Fournier, P. E. et al. Modern clinical microbiology: new challenges and solutions. Nat. Rev. Microbiol. 11, 574-585 (2013).

6. Cunningham, S. A. et al. Three-hour molecular detection of Campylobacter, salmonella, yersinia, and Shigella species in feces with accuracy as high as that of culture. J. Clin. Microbiol. 48, 2929-2933 (2010).

7. Mendes Silva, D. \& Domingues, L. On the track for an efficient detection of Escherichia coli in water: a review on PCR-based methods. Ecotoxicol. Environ. Saf. 113, 400-411 (2015)

8. Carey-Ann, B. D. \& Carroll, K. C. Diagnosis of Clostridium difficile infection: an ongoing conundrum for clinicians and for clinical laboratories. Clin. Microbiol. Rev. 26, 604-630 (2013).

9. Ahmed, A., Rushworth, J. V., Hirst, N. A. \& Millner, P. A. Biosensors for whole-cell bacterial detection. Clin. Microbiol. Rev. 27, 631-646 (2014).

10. Cao, J., Feng, C., Liu, Y., Wang, S. Y. \& Liu, F. Highly sensitive and rapid bacteria detection using molecular beacon-Au nanoparticles hybrid nanoprobes. Biosens. Bioelectron. 57, 133-138 (2014).

11. Jin, B. R. et al. Upconversion nanoparticles based FRET aptasensor for rapid and ultrasenstive bacteria detection. Biosens. Bioelectron. 90, 525-533 (2017).

12. Ma, X. Y., Song, L. J., Zhou, N. X., Xia, Y. \& Wang, Z. P. A novel aptasensor for the colorimetric detection of $S$. typhimurium based on gold nanoparticles. Int. J. Food Microbiol. 245, 1-5 (2017).

13. Verdoodt, N., Basso, C. R., Rossi, B. F. \& Pedrosa, V. A. Development of a rapid and sensitive immunosensor for the detection of bacteria. Food Chem. 221, 1792-1796 (2017). 
14. Thiramanas, R. \& Laocharoensuk, R. Competitive binding of polyethyleneimine-coated gold nanoparticles to enzymes and bacteria: a key mechanism for low-level colorimetric detection of gram-positive and gramnegative bacteria. Microchim Acta 183, 389-396 (2016).

15. Qi, P., Zhang, D., Zeng, Y. \& Wan, Y. Biosynthesis of CdS nanoparticles: a fluorescent sensor for sulfate-reducing bacteria detection. Talanta 147, 142-146 (2016).

16. Joo, J. et al. A facile and sensitive detection of pathogenic bacteria using magnetic nanoparticles and optical nanocrystal probes. Analyst 137 3609-3612 (2012).

17. Kumar, S. et al. A facile method for fabrication of buckled PDMS silver nanorod arrays as active 3D SERS cages for bacterial sensing. Chem. Commun. 51, 12411-12414 (2015).

18. Yang, L. L. et al. Engineering nanoparticle cluster arrays for bacterial biosensing: the role of the building block in multiscale SERS substrates. Adv. Funct. Mater. 20, 2619-2628 (2010).

19. Madiyar, F. R. et al. Integration of a nanostructured dielectrophoretic device and a surface-enhanced Raman probe for highly sensitive rapid bacteria detection. Nanoscale 7, 3726-3736 (2015).

20. Wang, C. W. et al. A rapid SERS method for label-free bacteria detection using polyethylenimine-modified Au-coated magnetic microspheres and Au@Ag nanoparticles. Analyst 141, 6226-6238 (2016).

21. Patel, I. S., Premasiri, W. R., Moir, D. T. \& Ziegler, L. D. Barcoding bacterial cells: a SERS-based methodology for pathogen identification. J. Raman Spectrosc. 39 1660-1672 (2008).

22. Wigginton, K. R. \& Vikesland, P. J. Gold-coated polycarbonate membrane filter for pathogen concentration and SERS-based detection. Analyst 135, 1320-1326 (2010).

23. McNeil, S. E. Characterization of Nanoparticles Intended for Drug Delivery. (Humana Press, New York, NY, 2011).

24. Park, B. S. et al. The structural basis of lipopolysaccharide recognition by the TLR4-MD-2 complex. Nature 458, 1191-1195 (2009).

25. Liu, T. Y. et al. Functionalized arrays of Raman-enhancing nanoparticles for capture and culture-free analysis of bacteria in human blood. Nat. Commun. $\mathbf{2}$ 538 (2011).

26. Oloomi, S. A. A., Saboonchi, A. \& Sedaghat, A. Effects of thin film thickness on emittance, reflectance and transmittance of nano scale multilayers. Int J. Phys. Sci. 5, 465-469 (2010).
27. Tezduyar, T. E. \& Sathe, S. Modelling of fluid-structure interactions with the space-time finite elements: solution techniques. Int J. Numer. Methods Fluids 54, 855-900 (2007).

28. Collin, S. Nanostructure arrays in free-space: optical properties and applications. Rep. Prog. Phys. 77, 126402 (2014).

29. Shaban, M., Hamdy, H., Shahin, F. \& Ryu, S. W. Optical properties of porous anodic alumina membrane uniformly decorated with ultra-thin porous gold nanoparticles arrays. J. Nanosci. Nanotechnol. 11, 941-952 (2011).

30. Im, H., Wittenberg, N. J., Lesuffleur, A., Lindquist, N. C. \& Oh, S. H. Membrane protein biosensing with plasmonic nanopore arrays and pore-spanning lipid membranes. Chem. Sci. 1, 688-696 (2010).

31. Mertens, J. et al. Controlling subnanometer gaps in plasmonic dimers using graphene. Nano. Lett. 13, 5033-5038 (2013).

32. Arcidiacono, S., Bieri, N. R., Poulikakos, D. \& Grigoropoulos, C. P. On the coalescence of gold nanoparticles. Int J. Multiph. Flow 30, 979-994 (2004).

33. Zhang, R. Y., Hummelgård, M. \& Olin, H. Single layer porous gold films grown at different temperatures. Phys. B Condens Matter 405, 4517-4522 (2010).

34. Pérez-González, O. et al. Optical spectroscopy of conductive junctions in plasmonic cavities. Nano. Lett. 10, 3090-3095 (2010).

35. Muhamadali, H. et al. Rapid, accurate, and comparative differentiation of clinically and industrially relevant microorganisms via multiple vibrational spectroscopic fingerprinting. Analyst 141, 5127-5136 (2016).

36. Bodelón, G. et al. Detection and imaging of quorum sensing in Pseudomonas aeruginosa biofilm communities by surface-enhanced resonance Raman scattering. Nat. Mater. 15, 1203-1211 (2016).

37. Matsushita, A. et al. Two-dimensional Fourier-transform Raman and nearinfrared correlation spectroscopy studies of poly(methyl methacrylate) blends: 1. Immiscible blends of poly(methyl methacrylate) and atactic polystyrene. Vib. Spectrosc. 24, 171-180 (2000).

38. Haiss, W., Thanh, N. T. K., Aveyard, J. \& Fernig, D. G. Determination of size and concentration of gold nanoparticles from UV-vis spectra. Anal. Chem. 79 4215-4221 (2007).

39. Johnson, P. B. \& Christy, R. W. Optical constants of the noble metals. Phys. Rev. B 6, 4370-4379 (1972). 